\title{
Comparison of Whole-Genome Sequences of Legionella pneumophila in Tap Water and in Clinical Strains, Flint, Michigan, USA, 2016
}

\author{
Emily Garner, Connor L. Brown, David Otto Schwake, William J. Rhoads, Gustavo Arango-Argoty, \\ Liqing Zhang, Guillaume Jospin, David A. Coil, Jonathan A. Eisen, Marc A. Edwards, Amy Pruden
}

During the water crisis in Flint, Michigan, USA (2014-2015), 2 outbreaks of Legionnaires' disease occurred in Genesee County, Michigan. We compared whole-genome sequences of 10 clinical Legionella pneumophila isolates submitted to a laboratory in Genesee County during the second outbreak with 103 water isolates collected the following year. We documented a genetically diverse range of $L$. pneumophila strains across clinical and water isolates. Isolates belonging to 1 clade ( 3 clinical isolates, 3 water isolates from a Flint hospital, 1 water isolate from a Flint residence, and the reference Paris strain) had a high degree of similarity (2-1,062 single-nucleotide polymorphisms), all $L$. pneumophila sequence type 1 , serogroup 1 . Serogroup 6 isolates belonging to sequence type 2518 were widespread in Flint hospital water samples but bore no resemblance to available clinical isolates. L. pneumophila strains in Flint tap water after the outbreaks were diverse and similar to some disease-causing strains.

$\mathrm{L}$ egionnaires' disease is a severe form of pneumonia caused by inhalation of virulent species of aerosolized Legionella bacteria. In January 2016, the Michigan Department of Health and Human Services (MDHHS) and the Genesee County Health Department publicly announced 2 Legionnaires' disease outbreaks in Genesee County, Michigan, USA $(1,2)$. The first outbreak occurred from June 2014 through March 2015 and the second from May 2015 through October 2015; a total of 90 cases and 12 deaths

Author affiliations: West Virginia University, Morgantown, West Virginia, USA (E. Garner); Virginia Polytechnic Institute and State University, Blacksburg, Virginia, USA (E. Garner, C.L. Brown, W.J. Rhoads, G. Arango-Argoty, L. Zhang, M.A. Edwards,

A. Pruden); Methodist University, Fayetteville, North Carolina, USA (D.O. Schwake); University of California at Davis, Davis, California, USA (G. Jospin, D.A. Coil, J.A. Eisen)

DOI: https://doi.org/10.3201/eid2511.181032 were documented (1-3). From April 2014 through October 2015, the city of Flint, in Genesee County, switched its drinking water source from Detroit Water and Sewer Department (DWSD), which used corrosion control, to the corrosive Flint River, without implementing federally mandated corrosion control; this new water source led to elevated lead in tap water over a prolonged period, now called the Flint water crisis (4). This disruption in water quality likely also stimulated the growth of L. pneumophila, the species most frequently identified as the causative agent of Legionnaires' disease (5,6), in Flint's distribution and plumbing systems (7).

Our prior work associated the Legionnaires' disease outbreaks with factors known to be conducive to Legionella growth: elevated iron (a consequence of corroded iron water mains), reduced free chlorine disinfectant residuals, and elevated water temperatures $(7,8)$. Later, Zahran and colleagues reported that the odds of Flint residents being referred for Legionnaires' disease treatment while the Flint River was the source of tap water increased 6.3fold and confirmed our report of associations with low chlorine residuals (9), but the odds analysis, which was based on the use of referral date rather than symptom onset date, excluded many healthcare-associated cases (10). Furthermore, during the second outbreak, Legionella spp. and L. pneumophila genes were found to be higher in the tap water of large buildings in Flint than in other water systems in US areas not experiencing outbreaks (8). Conversely, levels of the mip gene, which is specific to L. pneumophila, were largely below detection in Flint single-family residences, at least during the later stages of the water crisis when they were measured (2015-2016) (8). Large buildings with extensive plumbing networks, such as hospitals, are generally more susceptible to Legionella growth than are simpler plumbing systems characteristic of single-family homes (11); however, residences 
are also of interest for Legionella growth, given concerns about the high rate of sporadic Legionnaires' disease (12) and potential for exposure in the home.

Our study objective was to use next-generation DNA sequencing to compare L. pneumophila isolated from Flint tap water after the second Legionnaires' disease outbreak with tap water isolates from neighboring drinking water systems outside of Flint that were never served by Flint River water and clinical strains received during the second outbreak at a regional reference laboratory in Genesee County. Within Flint, Legionella isolates were obtained from the tap water of a hospital, a large public building, and single-family residences several months after the water source was switched back to DWSD. In addition to serogroup testing, we used whole-genome sequencing to compare isolates in terms of sequence type (ST), average nucleotide identity, and single-nucleotide polymorphisms (SNPs).

\section{Materials and Methods}

\section{Water Sample Collection and Legionella Isolation}

After Flint resumed purchasing water with corrosion control from the original supplier, DWSD, water sampling campaigns were conducted 5 months (March 7-9, 2016), 8 months (June 21-27, 2016), and 10 months (August 15$16,2016)$ later. Samples were collected from residences, small businesses, a large public building, and a hospital in Flint; as controls, samples were collected from buildings located outside of Flint that used DWSD or well water (Table 1). The March 2016 campaign targeted sampling of residences, small businesses, a large public building, and a hospital; samples were collected from hot (flushed for 30 seconds) and cold (stagnant) taps at each location. Samples were collected from the kitchen sink in homes and from restrooms in public buildings. The June 2016 campaign extensively sampled homes as part of a water heater cleaning campaign; the following samples were collected before and after a cleaning protocol: hot and cold stagnant kitchen tap samples, a stagnant shower sample of blended hot and cold water, a hot flushed kitchen tap sample, the water heater drain valve, and a flushed cold water sample from the outside hose bib or nearest tap to the service entry point. The August 2016 campaign targeted sampling from hot (flushed 30 seconds) and cold (stagnant) water taps from homes and small businesses. Legionella was cultured according to standard methods (13), and colonies were streaked to isolation.

\section{Clinical Isolates}

MDHHS provided 11 clinical isolates from de-identified Legionnaires' patients who received a diagnosis in 2015; however, 1 isolate could not be cultured and was deemed nonviable. When we initiated this study, we assumed that all 11 isolates originated from patients with some history of exposure in Flint or Genesee County during the Flint water crisis. However, we later learned that the commonality among clinical isolates was that they had been submitted to a Genesee County laboratory for analysis during the second outbreak and that 3 of the 11 isolates originated from patients who resided and received treatment outside of Genesee County (J. McFadden, MDHHS, pers. comm., $2017 \mathrm{Feb} 1$ ). Because the clinical isolates in this study were de-identified, comparison with the water isolates is described in terms of "L. pneumophila known to be capable of causing LD." We also included publicly available DNA sequence information from clinical reference strains in the analysis (Appendix 1 Table 2, https://wwwnc.cdc.gov/ EID/article/25/11/18-1032-App1.pdf).

\section{Whole-Genome Analysis of $L$. pneumophila Isolates}

Whole-genome sequencing was conducted by MicrobesNG (https://microbesng.uk) on an Illumina MiSeq (https://www. illumina.com) with $2 \times 250$-bp paired-end reads and Nextera library preparation (Illumina). Sequencing was performed for a representative subset of each building type and water source, including 103 water isolates and the 10 available clinical L. pneumophila isolates (Appendix 1 Table 1). To verify DNA integrity, DNA extracts were quantified via a Qubit 2.0 fluorometer (https://www.thermofisher.com) and analyzed via gel electrophoresis. Positive (L. pneumophila strain 130b) and negative (Stenotrophomonas maltophilia) control strains were also sequenced, and 3 clinical strains were sequenced in duplicate on $2 \mathrm{MiSeq}$ runs to evaluate run-to-run variation (Appendix 1 Figures 1-3). On average, 806,825 reads were obtained per isolate (range 280,380-2,031,828 reads). Reads were trimmed by using Trimmomatic (14), and de novo assemblies were generated by using SPAdes (15).

Genome sequences are available in GenBank under BioProject PRJNA453403. Legionella species assignments were determined via blastn (https://blast.ncbi.nlm.nih.gov) for isolate $16 \mathrm{~S}$ rRNA gene sequences. Average nucleotide identity was calculated as previously described (16), and SNPs were identified by using kSNP3.0 (17). We also included 9 L. pneumophila reference strains (Appendix 1 Table 2). We performed sequence-based typing targeting the flaA, pilE, asd, mip, mompS, proA, and neuA alleles (18) by using the mompS tool (19).

\section{Serogroup Analysis}

We identified $L$. pneumophila isolates belonging to serogroup 1 via detection of the wzm gene (20) in whole genome sequences. We verified DNA sequence-based classifications and determined unknown serogroups by using direct fluorescent antibody staining with fluorescein isothiocyanate-conjugated antibodies (m-TECH, http://www.4m-tech.com). 
Table 1. Total number of buildings sampled, number of samples collected, and number of isolates analyzed for Legionella, Flint, Michigan, USA*

\begin{tabular}{|c|c|c|c|c|c|c|c|c|c|}
\hline \multirow[b]{2}{*}{ Water sample source } & \multicolumn{3}{|c|}{ March 2016} & \multicolumn{3}{|c|}{ June 2016} & \multicolumn{3}{|c|}{ August 2016} \\
\hline & $\begin{array}{c}\text { No. } \\
\text { buildings } \\
\text { or samples }\end{array}$ & $\begin{array}{l}\text { No. (\%) } \\
\text { positive }\end{array}$ & $\begin{array}{c}\text { No. } \\
\text { isolates } \\
\text { analyzed }\end{array}$ & $\begin{array}{c}\text { No. } \\
\text { buildings } \\
\text { or samples }\end{array}$ & $\begin{array}{l}\text { No. (\%) } \\
\text { positive }\end{array}$ & $\begin{array}{c}\text { No. } \\
\text { isolates } \\
\text { analyzed }\end{array}$ & $\begin{array}{c}\text { No. } \\
\text { buildings } \\
\text { or samples }\end{array}$ & $\begin{array}{l}\text { No. }(\%) \\
\text { positive }\end{array}$ & $\begin{array}{c}\text { No. } \\
\text { isolates } \\
\text { analyzed }\end{array}$ \\
\hline Flint residences & 5 & 0 & & $32 \dagger$ & $2(6)$ & & $10 \ddagger$ & $2(20)$ & \\
\hline Hot (flushed) & 5 & 0 & 0 & 62 & $2(3)$ & 3 & 14 & $1(7)$ & 1 \\
\hline Hot (stagnant) & NS & & & 62 & $2(3)$ & 4 & NS & & \\
\hline Cold (flushed) & NS & & & 61 & $1(2)$ & 2 & NS & & \\
\hline Cold (stagnant) & 5 & 0 & 0 & 61 & $1(2)$ & 4 & 11 & $4(36)$ & 1 \\
\hline $\begin{array}{l}\text { Water heater } \\
\text { drain valve }\end{array}$ & NS & & & 62 & $1(2)$ & 5 & NS & & \\
\hline $\begin{array}{l}\text { Shower (hot and } \\
\text { cold) }\end{array}$ & NS & & & 62 & $1(2)$ & 2 & 3 & $1(33)$ & 1 \\
\hline Hospitals & 1 & $1(100)$ & & NS & & & NS & & \\
\hline Hot (flushed) & 19 & $16(84)$ & 56 & NS & & & NS & & \\
\hline Cold (stagnant) & 19 & $6(32)$ & 14 & NS & & & NS & & \\
\hline Buildings receiving & 4 & 0 & & NS & & & 8 & 0 & \\
\hline \multicolumn{10}{|l|}{ DWSD water } \\
\hline Hot (flushed) & 4 & 0 & 0 & NS & & & 8 & 0 & 0 \\
\hline Cold (stagnant) & 4 & 0 & 0 & NS & & & 8 & 0 & 0 \\
\hline Flint large buildings & 2 & $1(50)$ & & NS & & & NS & & \\
\hline Hot (flushed) & 5 & 0 & 0 & NS & & & NS & & \\
\hline Cold (stagnant) & 5 & $1(20)$ & 1 & NS & & & NS & & \\
\hline $\begin{array}{l}\text { Buildings receiving } \\
\text { well water }\end{array}$ & 1 & $1(100)$ & & NS & & & NS & & \\
\hline Hot (flushed) & 4 & $4(100)$ & $5 \S$ & NS & & & NS & & \\
\hline Cold (stagnant) & 3 & $2(67)$ & $4 \bar{\pi}$ & NS & & & NS & & \\
\hline Flint small businesses & 6 & 0 & & NS & & & 8 & 0 & \\
\hline Hot (flushed) & 6 & 0 & 0 & NS & & & 8 & 0 & 0 \\
\hline Cold (stagnant) & 6 & 0 & 0 & NS & & & 8 & 0 & 0 \\
\hline \multicolumn{10}{|c|}{$\begin{array}{l}\text { "Positive samples indicate presumptive } L \text {. pneumophila identified by performing culture according to the method described in (12). Unless otherwise } \\
\text { noted, identification as } L \text {. pneumophila was confirmed by using whole-genome sequencing. Boldface indicates total buildings sampled. Blank cells } \\
\text { indicate that data were not reported when applicable samples were not collected. NS, no samples of this type were collected. } \\
+1 \text { of the } 32 \text { homes was also sampled in March 2016. } \\
\neq 5 \text { of the } 10 \text { homes were also sampled in March 2016; } 1 \text { of the } 10 \text { was sampled in June } 2016 \text { (but not in March 2016; samples from this house were } \\
\text { positive on both dates). } \\
\$ 4 \text { of } 5 \text { isolates were a non-L. pneumophila species, according to whole-genome sequencing. } \\
\mathbb{I} 4 \text { of } 4 \text { isolates were a non- } L \text {. pneumophila species, according to whole-genome sequencing. }\end{array}$} \\
\hline
\end{tabular}

\section{Results}

\section{Legionella Isolate Characterization}

Of the 515 total water samples collected and from which $L$. pneumophila isolation was attempted (Table 1), 43 samples (8\%) were positive for Legionella. Of these, 22 (58\%) of 38 hospital samples from March 2016, eight (2\%) of 370 residence samples from June 2016 (positives originating from 2 separate residences), and 6 (21\%) of 28 residence samples from August 2016 (positives originating from different taps in a single residence) were positive for culturable $L$. pneumophila. No isolates were obtained from businesses receiving DWSD water, but $6(86 \%)$ of 7 taps at the school serviced by well water were identified as positive (although 5 of these were later determined to be Legionella species other than L. pneumophila).

16S rRNA genes mined from whole-genome sequences indicated that all clinical and water isolates, except for 8 of the 9 well water isolates, were L. pneumophila. The positive control strain was correctly identified as $L$. pneumophila; SNP analysis further classified it according to its known provenance (130b), and the negative control strain was also confirmed to be $S$. maltophilia (i.e., not Legionella). Serogrouping via presence of the wzm gene for serogroup 1 and direct fluorescent antibody staining for other serogroups indicated that all $L$. pneumophila isolates belonged to serogroups 1 and 6 (Table 2).

L. pneumophila isolates from clinical and water samples belonged to several STs (Table 2). Of serogroup 1 isolates, all belonged to STs 1, 44, 159, 192, 211, 213, or 222 or to a previously uncharacterized ST that we submitted to the European Working Group for Legionella Infections database (http://www.ewgli.org) and that has now been designated as ST2513. Serogroup 6 isolates all belonged to a previously uncharacterized ST that has now been designated as ST2518. Most hospital isolates belonged to ST2518, and isolates originating from residential tap water belonged primarily to ST192. Only ST1 was represented by both clinical and water isolates, specifically, 3 clinical isolates, 3 isolates from hospital tap water, and 1 isolate from residential tap water.

When classified according to SNP similarity, isolates formed distinct clades that were generally consistent with the ST classification (Figure). The ST1 clade varied by $2-1,062$ 
Table 2. Summary of Legionalla pneumophila isolates from Flint, Michigan, USA, 2016

\begin{tabular}{|c|c|c|}
\hline ST & Serogroup & Isolate origin* \\
\hline 1 & 1 & $\begin{array}{c}3 \text { hospital water }(\mathrm{HH} 17, \mathrm{HH} 25, \mathrm{HH} 56), 1 \\
\text { residence water (RH08), } 3 \text { clinical (C2, } \\
\text { C3, C7) }\end{array}$ \\
\hline 44 & 1 & 1 clinical (C6) \\
\hline 159 & 1 & 1 clinical (C1) \\
\hline 192 & 1 & $\begin{array}{l}19 \text { residence water (RC01, RC02, RC03, } \\
\text { RC04, RC06, RC07, RD01, RD02, RD03, } \\
\text { RD04, RD05, RH02, RH03, RH04, RH05, } \\
\text { RH07 RH07, RS01, RS02) }\end{array}$ \\
\hline 211 & 1 & 1 clinical (C8) \\
\hline 213 & 1 & 2 clinical $(\mathrm{C} 4, \mathrm{C} 5)$ \\
\hline 222 & 1 & 1 clinical (C9) \\
\hline $2513 \dagger$ & 1 & 1 clinical (C10) \\
\hline $2518 \dagger$ & 6 & 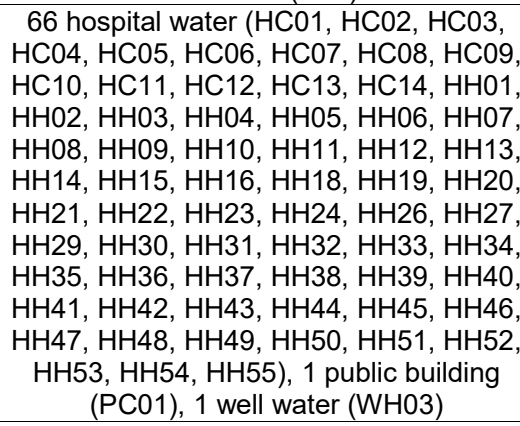 \\
\hline ND & ND & $\begin{array}{l}\text { HH28, RC05, RH01, RS03, WC01, WC02 } \\
\text { WC03, WC04, WH01, WH02, WH04, } \\
\text { WH05 }\end{array}$ \\
\hline
\end{tabular}

*First letter of isolate name indicates building type/location: $\mathrm{H}$, hospital; $\mathrm{R}$, residence; $\mathrm{W}$, school using well water; $\mathrm{P}$, large public building. Second letter indicates sample collection location; $\mathrm{H}$, hot water tap; $\mathrm{C}$, cold water tap; D, water heater drain valve; S, shower. Numerals $1-10$ indicate clinical strains. ND, not determined because of lack of $L$. pneumophilaspecific alleles or insufficient genome coverage; ST, sequence type. †New sequence types from this study submitted to European Working Group for Legionella Infections database (http://www.ewgli.org).

SNPs, and isolates varied from the reference Paris strain by 371-505 SNPs. In particular, clinical isolate C3 shared the highest degree of similarity with Flint tap water isolates (38-46 SNPs). Some degree of variation is expected to be associated with variability in sequencing accuracy because the 3 clinical isolates that were sequenced in duplicate on multiple MiSeq lanes differed from their replicate by $0-10$ SNPs. Several other distinct clades emerged in which water isolates were grouped primarily by building type. A large clade of ST2518 isolates included most samples from the hospital, 1 sample from well water, and 1 sample from a large public building. Another clade contained only isolates originating from Flint residence water samples belonging to ST192. The SNP results were confirmed by phylogenetic analysis and average nucleotide identity comparison (Appendix 1 Figures 1-3; Appendix 2, https://wwwnc.cdc.gov/ EID/article/25/11/18-1032-App2.xlsx).

The STs of 8 isolates derived from well water could not be determined because L. pneumophila-specific alleles were absent, suggesting that the isolates were mistakenly phenotypically characterized as L. pneumophila on the basis of colony morphology. Average nucleotide identity values comparing these isolates with the positive control L. pneumophila strain (130b) were 62.645\%-62.969\%, whereas average nucleotide identity values of a single species are generally $>95 \%$ (21). These 8 isolates seem to be most closely related to $L$. taurinensis, L. rubrilucens, or $L$. erythra, because the 16S rRNA genes extracted from these genomes shared $>99 \%$ nt similarity to all 3 species.

\section{Discussion}

When considered per capita, the Legionnaires' outbreaks in Genesee County are among the largest in US history. However, to our knowledge, few clinical sputum isolates were collected or preserved from these outbreaks; for most cases, only urine-antigen testing was conducted. A common problem in the United States is reliance on urine-antigen testing and lack of collection of clinical Legionella isolates; these practices unfortunately limit the ability to track sources of infection, learn from past outbreaks, and prevent future outbreaks $(22,23)$. Among the clinical sputum isolates that were sent to Genesee County laboratories during the outbreaks, none were from patients residing in homes serviced by Flint water (S. Lyon-Callo, MDHHS, pers. comm., 2018 Apr 5); thus, direct examination of potential residential exposure is not possible from this study. Given that $68 \%$ of patients' residences were confirmed to not have been serviced by Flint water (3), the potential exists that a portion of the remaining $32 \%$ had some residential exposure in Flint.

Another challenge of tracking sources of Legionnaires' disease is limited availability of water isolates. Given that the outbreaks were not publicly announced until 3 months after the conclusion of the second outbreak (January 2016), few environmental specimens were collected or preserved when the outbreaks were occurring. Analysis of any other water isolates that might exist from the time of the crisis would be valuable for learning more about this outbreak. MDHHS reported that 106 environmental Legionella specimens were retained at a Flint hospital but were not submitted to the State Health Department as had been requested (Sarah Lyon-Callo, MDHHS, pers. comm., 2019 Apr 30). Thus, a more definitive study of environmental sources of the outbreaks is not possible without a wider collection of clinical and environmental isolates.

Our study provides a survey of the landscape of genetic diversity among Legionella isolates collected from tap water from a range of building types served by the Flint drinking water distribution system over the 1-year period after the switch back to DWSD water. We compared these isolates with clinical isolates and with isolates from tap water of neighboring water systems never served by the Flint River or DWSD. Although it was not possible to collect water isolates during the actual outbreaks, previous studies have demonstrated that a single strain of 


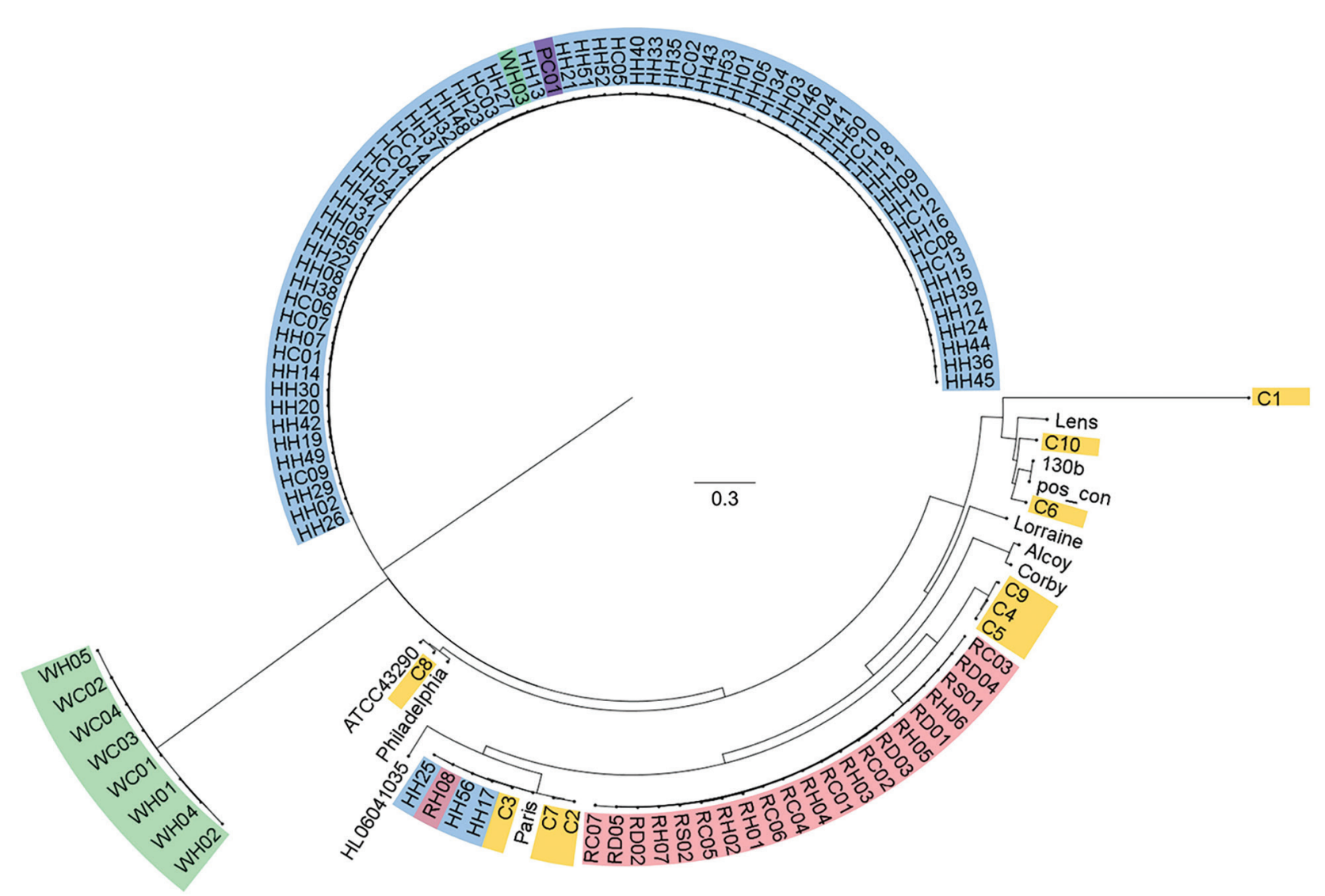

Figure. Single-nucleotide polymorphism (SNP) analysis of isolates from study of Legionella pneumophila in tap water, Flint, Michigan, USA. Analysis was conducted in kSNP3.0 (https://sourceforge.net/projects/ksnp/) and visualized by using FigTree 14.3 (https://github.com/rambaut/figtree/releases/tag/v1.4.3). Isolate sources: yellow, clinical samples; blue, hospital water; red, residence water; purple, public building water; green, buildings supplied by well water. With the exception of buildings supplied by well water, all buildings were serviced by Flint municipal water. Reference strains are detailed in Appendix 1 Table 2 (https://wwwnc.cdc.gov/EID/ article/25/11/18-1032-App1.pdf). Scale bar indicates nucleotide substitutions per site.

L. pneumophila can colonize buildings and persist over multiple years (24-26). Thus, it is reasonable to assume that water isolates collected in 2016 were probably representative of strains colonizing building water systems over the previous months or even years.

Our study provides reasonable evidence that plumbing served by the Flint drinking water system was colonized by strains of L. pneumophila capable of causing Legionnaires' disease, particularly serogroup 1 and ST1. Although no epidemiologic links have been made between clinical cases and cooling tower exposures in these outbreaks, direct or indirect use of tap water (e.g., via feed to cooling towers) is possible. High degrees of similarity (2-1,062 SNPs) were noted between the ST1 isolates of clinical and water origin, a finding that was consistent with phylogenetic and average nucleotide identity analysis (Appendix 1 Figures 1-3; Appendix 2). The highest degree of similarity between clinical and water isolates was between $\mathrm{C} 3$ and RH08 (38 SNPs), HH25 (40 SNPs), HH17 (45 SNPs), and HH56 (46 SNPs). C2 differed from water isolates by $1,053-1,062$ SNPs, and
C7 differed from water isolates by 1,041-1,049 SNPs. With the exception of 1 isolate (WH03), isolates obtained from tap water from buildings never served by the Flint River were markedly distinct from those originating from residences or hospitals in Flint as well as the clinical isolates. The low number of SNPs between replicate genomes sequenced in this study $(0-10)$ suggests that the extent to which technical variation in whole-genome sequencing contributed to observed sequence variation is low. Previous studies have documented that although some Legionnaires' disease outbreaks are characterized by $L$. pneumophila clinical strains that differ by as few as $<5$ SNPs, other outbreaks may differ by as many as 418 core SNPs (27). Thus, the SNP variability between water and clinical strains of ST1 in this study, particularly C3, is comparable to the documented range of variation in other outbreaks. In contrast, clinical strain $\mathrm{C} 2$ varied from the Paris reference strain by only $505, \mathrm{C} 3$ by 371 , and C7 by 491 SNPs. Therefore, ascertaining what level of SNP divergence between strains is demonstrative of a common source or virulent strain is 
challenging. Given the well-established pathogenicity of the Paris strain, the results are also suggestive of genomic similarity among virulent strains of Legionella. Regardless, the similarity between $\mathrm{C} 3$ and strains isolated from Flint tap water samples (38-46 SNPs) is notable.

ST1 water isolates were collected from taps of a hospital and a residence, indicating that this ST seems to have been somewhat widespread in the water distribution system, spanning multiple Flint buildings. However, the presence of several distinct phylogenetic clades of L. pneumophila isolated from Flint water systems further demonstrates that a single strain of $L$. pneumophila did not dominate the system citywide. We hypothesize that this finding is likely the result of conditions favorable to Legionella growth, which we previously documented in the Flint system (7), facilitating the proliferation of multiple strains of L. pneumophila in different buildings and parts of the system. Similarly, the broad distribution of clinical isolates across 7 STs supports the hypothesis that any waterborne exposures that resulted in Legionnaires' disease could hypothetically have originated from a diverse array of $L$. pneumophila strains and exposure sources.

All clinical isolates characterized in this study belonged to L. pneumophila serogroup 1, which is identified as the cause of $\geq 57 \%$ of reported Legionnaires' cases in the United States (6). ST1 (belonging to serogroup 1) has been widely implicated in Legionnaires' outbreaks worldwide, including outbreaks in France (28), China (29), Germany (30), Canada (31), and the United States (32). In the United States, ST1 is thought to be both the most common cause of sporadic Legionnaires' disease cases and the most common waterborne ST found in potable and nonpotable water (32). ST1 isolates are highly conserved at the nucleotide level (33), making it challenging to link clinical cases with environmental sources because of the prevalence of ST1 and lack of genetic variability.

Water isolates belonging to serogroup 6, all classified as ST2518, were widespread in samples collected from a Flint hospital in March 2016. A study of L. pneumophila isolates collected from Flint tap water in September and October 2016 also found that serogroup 6 isolates were widespread in residential premise plumbing water samples, although these isolates all belonged to STs 367 and 461 (34). Byrne and colleagues found that serogroup 6 strains were at least as infectious for macrophages as a known virulent laboratory strain, emphasizing the potential for Legionnaires' disease to be caused by strains other than serogroup 1 (34), although more research is needed to confirm the relevance of serogroup 6 strains for human infectivity. In our study, none of the clinical strains available for analysis were serogroup 6 .

It is noteworthy that $19 \%$ of hot water and $12 \%$ of cold water taps were positive for culturable L. pneumophila.
Although L. pneumophila typically multiplies at $25^{\circ} \mathrm{C}-$ $37^{\circ} \mathrm{C} \mathrm{(35)} \mathrm{and} \mathrm{prospers} \mathrm{in} \mathrm{hot} \mathrm{water} \mathrm{plumbing} \mathrm{systems} \mathrm{(36),}$ it has also been widely documented in cold water taps; one molecular analysis-based study found that as many as $47 \%$ of surveyed cold water taps were positive for genes specific to L. pneumophila serogroup 1 (37).

When MDHHS recently conducted an epidemiologic characterization of the Genesee County Legionnaires' disease cases recorded in 2014 and 2015, although a lack of clinical isolates hampered a comprehensive investigation, they found that exposures that occurred at 1 Flint hospital potentially explained most cases $(1-3)$. Our study provides complementary whole-genome sequencing-based characterization of clinical isolates and tap water L. pneumophila isolates collected after the Flint outbreaks. Notably, we found a high degree of similarity between 4 water isolates originating from Flint tap water and 3 regional clinical strains known to cause Legionnaires' disease. Our study also established that a variety of $L$. pneumophila strains were culturable from Flint tap water and that they tended to cluster genetically by residence versus hospital origin. Likewise, we found notable diversity among clinical strains, spanning 7 STs. Thus, multiple L. pneumophila strains were associated with the Flint 2014-2015 Legionnaires' outbreaks, potentially resulting from multiple sources of exposure, although further epidemiologic investigation is needed to identify whether multiple sources were involved and whether there were any common sources of exposure. Although we did not intend for this study to provide an epidemiologic analysis of precise sources of Legionella exposure for Legionnaires' patients, our publicly available data could support such studies in the future.

\section{Acknowledgments}

We thank the members of the Flint Water Study Team at Virginia Polytechnic Institute and State University who volunteered their time to collect samples and the Flint citizens and businesses that supported this study. We thank Joan Rose for providing access to the laboratory at Michigan State University to support the fieldwork.

This study was supported by the US National Science Foundation (RAPID award no. 1556258), a Graduate Research Fellowship Program Grant (DGE 0822220), and supplementary funding (associated with award no. 1336650). Additional support was provided by the Alfred P. Sloan Foundation Microbiology of the Built Environment program, the Michigan Department of Environmental Quality for a study of effects of flushing residential hot water heaters (June 2016), the American Water Works Association Abel Wolman Fellowship, and the Institute for Critical Technology and Applied Science and Engineering of the Exposome Center at Virginia Polytechnic Institute and State University. 
In addition to the funding sources noted, author M.A.E. contributed discretionary research funding to support this study. No funds were received from the State of Michigan or MDHHS for this study. M.A.E. was subpoenaed to testify by the defense in the felony case against MDHHS employees Eden Wells and Nick Lyon in March 2018 and did so without compensation. In addition, coauthors M.A.E. and A.P. were principal investigators on a $\$ 263,000$ subcontract to the National Sanitation Foundation on a project funded by the State of Michigan to study point-ofuse filters, but this project was canceled by the National Sanitation Foundation midway through the study without any samples having been collected or processed.

\section{About the Author}

Dr. Garner is an assistant professor of civil and environmental engineering at West Virginia University. Her research focuses on the dissemination of opportunistic pathogens and antibiotic-resistant bacteria in water systems, with a particular emphasis on water sustainability and water reuse.

\section{References}

1. Michigan Department of Health and Human Services. Legionellosis outbreak - Genesee County, June, 2014-March, 2015 full analysis. 2016 [cited 2018 Jun 22]. https://www.michigan.gov/ documents/mdhhs/6-14_to_3-15_Legionellosis_Report_Full_ Analysis_Results_51170죽.pdf

2. Michigan Department of Health and Human Services. Legionellosis outbreak - Genesee County, May 2015-October 2015 full analysis. 2016 [cited 2018 Jun 22]. https://gchd.us/ wp-content/uploads/2016/08/Updated-LD-Report-4-11-16.pdf

3. Michigan Department of Health and Human Services. Epidemiology of Legionnaires' disease in Genesee County, Michigan, 2014-2017 chart book. 2018 [cited 2018 Jun 22]. https://content.govdelivery.com/attachments/MIDHHS/2018/05/29/ file_attachments/1014713/GC\%2BLegionella\%2BChartBook\%2B FINAL.pdf

4. Pieper KJ, Martin R, Tang M, Walters L, Parks J, Roy S, et al. Evaluating lead in water levels during the Flint Water Crisis and associated remediation interventions. Environ Sci Technol. 2017;52:8124-32. https://doi.org/10.1021/acs.est.8b00791

5. Dooling KL, Toews K, Hicks LA, Garrison LE, Bachaus B, Zansky S. Active Bacterial Core Surveillance for LegionellosisUnited States, 2011-2013. MMWR Morb Mortal Wkly Rep. 2015;64:2011-3.

6. Marston BJ, Lipman HB, Breiman RF. Surveillance for Legionnaires' disease. Risk factors for morbidity and mortality. Arch Intern Med. 1994;154:2417-22. https://doi.org/10.1001/ archinte.1994.00420210049006

7. Rhoads WJ, Garner E, Ji P, Zhu N, Parks J, Schwake DO, et al. Distribution system operational deficiencies coincide with reported Legionnaires' disease clusters in Flint, Michigan. Environ Sci Technol. 2017;51:11986-95. https://doi.org/10.1021/acs. est. $7 \mathrm{~b} 01589$

8. Schwake DO, Garner E, Strom OR, Pruden A, Edwards MA. Legionella DNA markers in tap water coincident with a spike in Legionnaires' disease in Flint, MI. Environ Sci Technol Lett. 2016;3:311-5. https://doi.org/10.1021/acs.estlett.6b00192

9. Zahran S, McElmurry SP, Kilgore PE, Mushinski D, Press J, Love NG, et al. Assessment of the Legionnaires' disease outbreak in Flint, Michigan. Proc Natl Acad Sci U S A. 2018;115:E1730-9. https://doi.org/10.1073/pnas.1718679115

10. Michigan Department of Health and Human Services. MDHHS response to Flint Area Community Health and Environment Partnership, Proceedings of the National Academy of Sciences Article. February 5, 2018 [cited 2018 Jun 22]. https://www.michigan.gov/documents/mdhhs/MDHHS_Response_ to_FACHEP_Proceedings_of_the_National_Academy_of Sciences_Article_FINAL_613088_7.pdf

11. Sabria M, Yu VL. Hospital-acquired legionellosis: solutions for a preventable infection. Lancet Infect Dis. 2002;2:368-73. https://doi.org/10.1016/S1473-3099(02)00291-8

12. Hicks LA, Garrison LE, Nelson GE, Hampton LM; Centers for Disease Control and Prevention (CDC). LegionellosisUnited States, 2000-2009. MMWR Morb Mortal Wkly Rep. 2011;60:1083-6.

13. Centers for Disease Control and Prevention, Procedures for the recovery of Legionella from the environment [cited 2018 Jun 22]. https:/www.epa.gov/sites/production/files/2018-11/documents/ cdc_legionella_method.pdf

14. Bolger AM, Lohse M, Usadel B. Trimmomatic: a flexible trimmer for Illumina sequence data. Bioinformatics. 2014;30:2114-20. https://doi.org/10.1093/bioinformatics/btu170

15. Bankevich A, Nurk S, Antipov D, Gurevich AA, Dvorkin M, Kulikov AS, et al. SPAdes: a new genome assembly algorithm and its applications to single-cell sequencing. J Comput Biol. 2012;19:455-77. https://doi.org/10.1089/cmb.2012.0021

16. Goris J, Konstantinidis KT, Klappenbach JA, Coenye T, Vandamme P, Tiedje JM. DNA-DNA hybridization values and their relationship to whole-genome sequence similarities. 2007; 57:81-91.

17. Gardner SN, Slezak T, Hall BG. kSNP3.0: SNP detection and phylogenetic analysis of genomes without genome alignment or reference genome. Bioinformatics. 2015;31:2877-8. https://doi.org/10.1093/bioinformatics/btv271

18. Gaia V, Fry NK, Afshar B, Lu PC, Etienne J, Peduzzi R, et al. Consensus sequence-based scheme for epidemiological typing of clinical and environmental isolates of Legionella pneumophila. J Clin Microbiol. 2005;43:2047-52.

19. Gordon M, Yakunin E, Valinsky L, Chalifa-Caspi V, Moran-Gilad J; ESCMID Study Group for Legionella Infections. A bioinformatics tool for ensuring the backwards compatibility of Legionella pneumophila typing in the genomic era. Clin Microbiol Infect. 2017;23:306-10. https://doi.org/10.1016/j.cmi.2017.01.002

20. Mérault N, Rusniok C, Jarraud S, Gomez-Valero L, Cazalet C, Marin M, et al.; DELPH-I Study Group. Specific real-time PCR for simultaneous detection and identification of Legionella pneumophila serogroup 1 in water and clinical samples. Appl Environ Microbiol. 2011;77:1708-17. https://doi.org/10.1128/ AEM.02261-10

21. Rodriguez-R LM, Konstantinidis KT. Bypassing cultivation to identify bacterial species: culture-independent genomic approaches identify credibly distinct clusters, avoid cultivation bias, and provide true insights into microbial species [cited 2019 Sep 4]. https://www.asmscience.org/content/journal/microbe/10.1128/ microbe.9.111.1

22. Berkelman RL, Pruden A. Prevention of Legionnaires' disease in the 21 st century by advancing science and public health practice. Emerg Infect Dis. 2017;23:1905-7. https://doi.org/10.3201/ eid2311.171429

23. Phin N, Parry-Ford F, Harrison T, Stagg HR, Zhang N, Kumar K, et al. Epidemiology and clinical management of Legionnaires' disease. Lancet Infect Dis. 2014;14:1011-21. https://doi. org/10.1016/S1473-3099(14)70713-3

24. Perola O, Kauppinen J, Kusnetsov J, Kärkkäinen UM, Lück PC, Katila ML. Persistent Legionella pneumophila colonization of a 
hospital water supply: efficacy of control methods and a molecular epidemiological analysis. APMIS. 2005;113:45-53. https://doi.org/ 10.1111/j.1600-0463.2005.apm1130107.x

25. Rangel-Frausto MS, Rhomberg P, Hollis RJ, Pfaller MA, Wenzel RP, Helms CM, et al. Persistence of Legionella pneumophila in a hospital's water system: a 13-year survey. Infect Control Hosp Epidemiol. 1999;20:793-7. https://doi.org/ $10.1086 / 501586$

26. Scaturro M, Fontana S, Dell'eva I, Helfer F, Marchio M, Stefanetti MV, et al. A multicenter study of viable PCR using propidium monoazide to detect Legionella in water samples. Diagn Microbiol Infect Dis. 2016;85:283-8. https://doi.org/10.1016 j.diagmicrobio.2016.04.009

27. Raphael BH, Baker DJ, Nazarian E, Lapierre P, Bopp D, Kozak-muiznieks NA, et al. Genomic resolution of outbreakassociated Legionella pneumophila serogroup 1 isolates from New York State. Appl Environ Microbiol. 2016;82:3582-90.

28. Ginevra C, Jacotin N, Diancourt L, Guigon G, Arquilliere R, Meugnier $\mathrm{H}$, et al. Legionella pneumophila sequence type 1/ Paris pulsotype subtyping by spoligotyping. J Clin Microbiol. 2012;50:696-701. https://doi.org/10.1128/JCM.06180-11

29. Qin T, Zhang W, Liu W, Zhou H, Ren H, Shao Z, et al. Population structure and minimum core genome typing of Legionella pneumophila. Sci Rep. 2016;6:21356. https://doi.org/10.1038/srep21356

30. Borchardt J, Helbig JH, Lück PC. Occurrence and distribution of sequence types among Legionella pneumophila strains isolated from patients in Germany: common features and differences to other regions of the world. Eur J Clin Microbiol Infect Dis. 2008;27:29-36. https://doi.org/10.1007/s10096-007-0392-3

31. Reimer AR, Au S, Schindle S, Bernard KA. Legionella pneumophila monoclonal antibody subgroups and DNA sequence types isolated in Canada between 1981 and 2009: Laboratory Component of National Surveillance. Eur J Clin Microbiol Infect Dis. 2010;29:191-205. https://doi.org/10.1007/s10096-009-0840-3

32. Kozak-Muiznieks NA, Morrison SS, Mercante JW, Ishaq MK, Johnson T, Caravas J, et al. Comparative genome analysis reveals a complex population structure of Legionella pneumophila subspecies. Infect Genet Evol. 2018;59:172-85. https://doi.org/ 10.1016/j.meegid.2018.02.008

33. Mercante JW, Caravas JA, Ishaq MK, Kozak-Muznieks NA, Raphael BH, Winchell JM. Genomic heterogeneity differentiates clinical and environmental subgroups of Legionella pneumophila sequence type 1. PLoS One. 2018;13:e0206110

34. Byrne BG, McColm S, McElmurry SP, Kilgore PE, Sobeck J, Sadler R, et al. Prevalence of infection-competent serogroup 6 Legionella pneumophila within premise plumbing in southeast Michigan. MBio. 2018;9:e00016-8. https://doi.org/10.1128/ mBio.00016-18

35. Wadowsky RM, Wolford R, McNamara AM, Yee RB. Effect of temperature, $\mathrm{pH}$, and oxygen level on the multiplication of naturally occurring Legionella pneumophila in potable water. Appl Environ Microbiol. 1985;49:1197-205.

36. Rhoads WJ, Ji P, Pruden A, Edwards MA. Water heater temperature set point and water use patterns influence Legionella pneumophila and associated microorganisms at the tap. Microbiome. 2015;3:1-13.

37. Donohue MJ, O'Connell K, Vesper SJ, Mistry JH, King D, Kostich M, et al. Widespread molecular detection of Legionella pneumophila serogroup 1 in cold water taps across the United States. Environ Sci Technol. 2014;48:3145-52. https://doi.org/ $10.1021 /$ es4055115

Address for correspondence: Amy Pruden, Virginia Polytechnic Institute and State University, Civil and Environmental Engineering, 418 Durham Hall, 1145 Perry St, Blacksburg, VA 24061-0131, USA; email: apruden@vt.edu
EID Podcast Developing Biological Reference Materials to Prepare for Epidemics

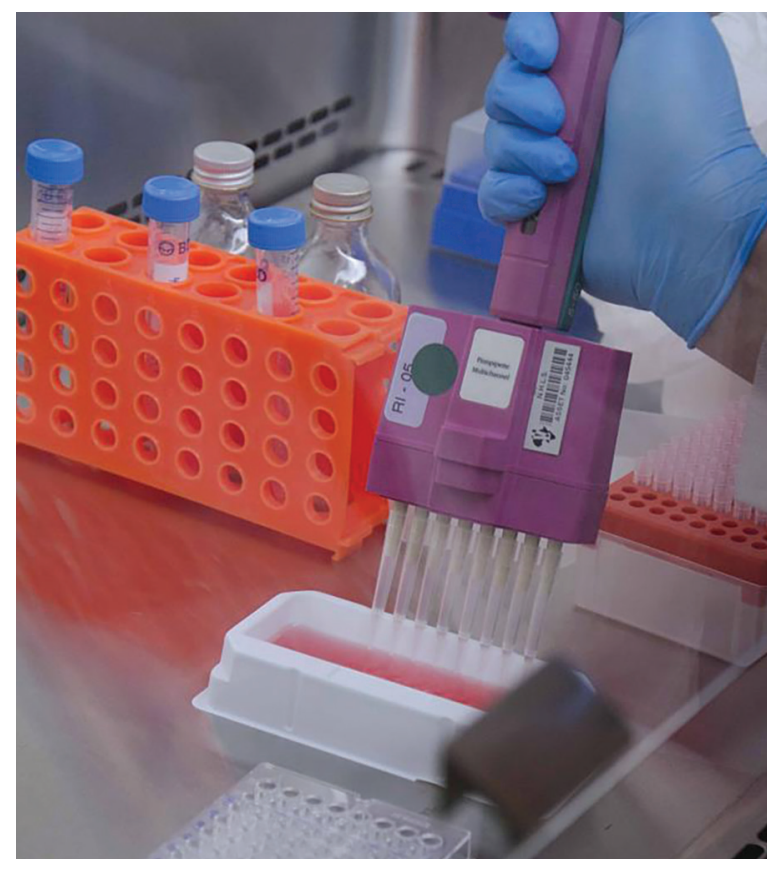

Having standard biological reference materials, such as antigens and antibodies, is crucial for developing comparable research across international institutions. However, the process of developing a standard can be long and difficult.

In this EID podcast, Dr. Tommy Rampling, a clinician and academic fellow at the Hospital for Tropical Diseases and University College in London, explains the intricacies behind the development and distribution of biological reference materials.

\section{Visit our website to listen:} https: //go.usa.gov/xyfJX

\section{EMERGING INFECTIOUS DISEASES}

